M. Jaschek and D. Egret

Observatoire de Strasbourg

We are presently undertaking, at the Stellar Data Center, a large effort to produce the Catalogue of Stellar Groups, which is a catalogue listing all the kinds of stars with spectral peculiarities existing up to now. The work is in progress and presently about fifty groups (Ap, $\mathrm{Am}, \mathrm{Be}, \mathrm{WR}, \mathrm{H}$ and $\mathrm{K}$ emission 1 ine stars, etc.) are defined, gathering. more than 25000 stars. The catalogue was started with the aim to set up lists defined by homogeneous criteria, because up to now the available information is scattered through the literature. We hope that the catalogue will be useful for formulating observing programs and for further detailed research on each of the groups.

For this symposium we have compiled a list of Be stars and prepared a comprehensive catalogue of Be stars on microfiche.

\title{
1. DEFINITION
}

The definition of a Be star we accepted is that of a non-supergiant B star which showed emission in one Balmer line at least once. For this reason we have excluded, with one exception, all stars for which no MK classification exists. The exception is that of Bidelman and MacConnell (1973) who used the same definition but provided no MK type. (Note that only 132 stars come from this 1 ist).

Besides supergiants, also planetary nebulae and spectroscopic binaries were excluded. The $B[e]$ stars are 1 isted as a separate table. These stars are defined as being those showing proper emissions of $\mathrm{Be}$ stars plus emission of some forbidden line.

The catalogue contains about 1100 stars. From confrontation with the number of stars in our catalogue group "H $\alpha$ emission stars", which includes about 5000 stars, it is evident that we have made a strong selection. On the contrary, one can be reasonably sure that the stars in our catalogue are authentic Be stars.

Please notice that MK types are established in a wavelength region in which $\mathrm{H} \alpha$ is not included. Therefore many stars having $\mathrm{H} \alpha$ in emission are classified as normal stars, being called for instance B3 $\mathrm{V}$ and not B3 $\mathrm{V}$ e. 


\section{THE DATA}

The final catalogue can be considered as the intersection of three main files: the lists of emission-line stars, the compilations or lists of MK classifications and the data base of the Stellar Data Center.

Our major source for emission line stars was the "Catalogue of Stars Whose Spectra have shown Emission Lines" (Wackerling, 1970). We also used the 1ist by Bidelman and MacConnel1 (1973), and some additional stars were found in the lists of MK classifications.

Our sources for the MK classifications are the "Catalogue of Selected Spectral Types" (Jaschek, 1978), the Michigan Spectral Survey for HD stars, vo1. I and II (Houk and Cowley, 1975, Houk, 1978), the compilation by Uesugi (1976) and the last edition of the MK Extension (Kennedy, 1980). Other classifications by Lesh (1968), Graham (1970), Henize (1976), Andersen and Nordström (1977, 1979) and Jaschek et al. (1980) are used.

In a final step we have searched for each star the fundamental data and measures available in the Catalogue of Stellar Identifications (Ochsenbein et al., 1981). The following data are listed in the microfiche edition of the catalogue: $\mathrm{DM}$ and $\mathrm{HD}$ identification, number in the main 1ists of emission line stars (MWC, AS, Henize), coordinates, V magnitude and UBV photometry (when available from Nicolet's compilation, 1978), adopted MK classification, radial velocity, rotational velocity; an asterisk indicates when the star is known as a visual binary or a spectroscopic binary, and if the star is measured in other photometric systems (uvby, infrared and ultraviolet); if the star is known as a variable star, the name is given.

The list of $B$ [e] stars is given in a separate table.

A bibliographical file for the same stars is also in preparation: we have used the Bibliographical Star Index (Spite et a1., 1980) to obtain a list of the papers (complete reference and title) in which the star is quoted, in the last ten years (since 1970, date of the preparation of the bibliographical catalogue by Jaschek et a1., 1971). This 1 ist will be published as a separate microfiche.

\section{FINAL REMARKS}

A first analysis of this catalogue is presented in the first session of this symposium (Egret, 1982).

The microfiche can be requested from the Centre de Données Stellaires (Observatoire de Strasbourg).

\section{REFERENCES}

Andersen, J. and Nordström, B.: 1977, Astron.Astrophys.Supp1.Ser. 29,309. Andersen,J. and Nordström,B.: 1979, Inf.Bul1.C.D.S. 15,39. Bidelman,W.P. and MacConne11,D.,J.: 1973, Astron.J. 78,687. Egret,D.: 1982, IAU Symp.98 Be Stars, Jaschek,M. and Groth,H.G. eds., 23 Graham,J.,A.: 1970, Astron.J. 75,703. 
Henize,K.: 1976, Astrophys.J.Suppl.Ser. 30,491.

Houk,N. and Cowley,A.,P.: 1975, Michigan Catalogue of 2-dimensional Spectral Types for the HD Stars, vol.I, Univ. Michigan.

Houk, N.: 1978, Michigan Catalogue of 2-dimensional Spectral Types for the HD Stars, vol.II, Univ. Michigan.

Jaschek,M.: 1978, Inf.Bul1.C.D.S. 15,121.

Jaschek,C.,Ferrer,L., Jaschek,M.: 1971, La P1ata Obs., Ser.Astron.,XXXVII.

Jaschek, M. ,Hubert-Delplace,A.-M., Hubert,H., Jaschek, . : 1980, Astron. Astrophys.Supp1.Ser. 42,103.

Kennedy,P.,M.: 1980, MK Classification Extension, MtStromlo Obs.

Lesh,J.,R.: 1968, Astrophys.J.Supp1.Ser. 17,371.

Nicolet,B.: 1978, Astron.Astrophys.Supp1.Ser. 34,1.

Ochsenbein,F.,Bischoff,M.,Egret,D.: 1981, Astron.Astrophys.Supp1.Ser. 43,259 .

Spite,F.,Ochsenbein,F.,Kirchner,S.,Lahmek,R.: 1980, Inf.Bu11.C.D.S. 18, 89.

Uesugi,A.: 1976, A Revised Catalogue of Stellar Rotational Velocities, preliminary edition, preprint.

Wackerling,L.,R.: 1970, Mem.Roy.Astron.Soc. 73,153.

\section{DISCUSSION}

Andrillat: Dans la définition que vous avez adoptée pour les étoiles Be, vous indiquez qu'il s'agit d'étoiles ayant montré une fois $\mathrm{H}_{\alpha}$ en émission : à partir de quelle date?

Egret: The oldest observations of $\mathrm{H}_{\alpha}$ in emission we used are those compiled by Wackerling (1970) (including the Mt. Wilson catalogue, etc.). 